\title{
Text to Multiple Language Translator
}

\author{
Deepak kumar \\ Department of Information Technology, Delhi, India \\ To Cite this Article \\ Deepak kumar, "Text to Multiple Language Translator", International Journal for Modern Trends in Science and \\ Technology, 6(12): 411-413, 2020.

\section{Article Info} \\ Received on 16-November-2020, Revised on 09-December-2020, Accepted on 12-December-2020, Published on 18-December-2020.
}

\section{ABSTRACT}

In today's globalized world thousands of peoples travels from one country to another. To interact with different peoples we generally speaks in English and communicate but this can't be possible always as sometimes we need to talk in the local language so for these situations my system comes in picture as is provides quick, simple, and reliable way to convert a sentence into many language. Not only convert but also speaks so that user don't need to speak in local language and can easily communicate.

\section{INTRODUCTION}

This system is a Multilanguage translator which converts the given text into an audio output and audio to text and text to text as well. It supports multiple language translation from English to language selected by the user. To use this system user just need to type the text and fix the rate and pitch of the output according to their convenience. This system automatically set tones according to the text provided by the user i.e "how are you I am good" and "how are you , I am good " the system will speaks both the strings differently.

Here we use vanilla js with web speech api at back end which process the user data and convert into the audio message.

Speech synthesis is accessed via the Speech Synthesis interface, a text-to-speech component that allows programs to read out their text content (normally via the device's default speech synthesizer .) Different voice types are represented by Speech Synthesis Voice objects, and different parts of text that you want to be spoken are represented by Speech Synthesis Utterance objects. You can get these spoken by passing them to the Speech_Synthesis.speak() method.
Speech-To-Text, Text-To-Speech technology. As all these functionalities and services for the program have been explained, the main structure and construction of the project has been basically illustrated with its goals.

Apart from the project itself, there is also some investigation works on the existed products in this area and the tendency of the voice product, personal assistant developing. Products were mainly investigated that are popular and representative, the Googles product of "google translator". The main focus behind this investigation was to determine how those ideas originated; what functionalities and services they have; how they provide these services to the customers; test the product and related functions to get the architect, structure, logical algorithms of those products; how they spread and promote in marketing and how they refine and upgrade the products from different versions.

\section{RELATED WORK}

Googles google translator is now a signature feature of machines. Mainly controlled by a user's voice, the digital assistant's primary ability to 
understand when its spoken to and understanding what's said is important.

\section{Google translator}

Google Translate is developed by google was a multi language neural machine translation service, it is used to translate websites and texts from one language to another language. It used on website interface, mobile app for Android and IOS, and an application programming interface that helps developers build browser extensions and software applications. As of October 2020, Google Translate supports 108 languages at various levels and as of March 2016, claimed over 500+ million total users, with $100+$ billion words translated daily.

Google Translate produces across languages of multiple forms of text and media, including speech, text, websites, or text on display in still or live video images. In languages Google Translator can synthesize speech from text, and in some pairs it is possible to highlight the specific corresponding phrases and words between the source and target text. Results are sometimes shown with information below the translation box, but it is not a dictionary and has been shown to invent translation in all languages for words it does not recognize. If selected language detects unknown text language can be automatically identified. In the web interface, users can suggest different translations, such as for technical terms, or correct mistakes. To the translation process all these suggestions can be included in future updates. Google translator will produce a hyperlink to websites machine translation if user will enters a URL in the text. For later uses client can save proposals of translation in a 'phrasebook'. For some languages, text can be entered via an on-screen keyboard, through handwriting recognition, or speech recognition. It is possible to enter searches in a source language that are first translated to a destination language allowing one to browse and interpret results from the selected destination language in the source language.

On April 26, 2011, Google announced that the Google Translator API for software developers has been deprecate and would cease functioning.The Translate API page stated the reason as 'substantial economic burden caused due to extensive abuse' with an end date set for October 1, 2011. In response to public pressure, Google announced in July 2011 that the API would continue to be available as a paid service.

As the API was used in numerous third-party websites and apps, the original decision to deprecate it led some developers to criticize Google and question the viability of using Google APIs in their products.

In May 2006, Google launch google translator with a statistical translation machine engine.

Since translators algorithms was based on statistical or pattern analysis google translate does not apply grammatical rules rather than traditional rule-based analysis. The application original creator, Franz Josef Och, has criticized the effectiveness of rule-based algorithms in favor of statistical approaches. Original versions of Google Translate were based on a method called statistical machine translation, and more specifically, on research Google Translate does not apply grammatical rules by Och who won the darp contest for fast machine translation in 2003. Och was the head of Google's translation group until leaving to join Human Longevity, Inc. in Aug 2014.

Google Translator does not translate from one language to another $(11 \rightarrow 12)$. Instead, it often translates first to English and then to the target language $[11 \rightarrow \mathrm{eg} \rightarrow 12]$. However, because English, like all human languages, are ambiguous and depends on context, this can cause translation errors. For example, translating vous from French to Russian gives vous $\rightarrow$ you $\rightarrow$ tb 1 OR bb1/bb1. If Google were using an unambiguous, artificial language as the intermediary, it would be vous $\rightarrow$ you $\rightarrow$ bb1/bb1 OR tu $\rightarrow$ thou $\rightarrow$ tb 1 . Such a suffixing of words unambiguates their different possible meanings. Therefore publishing in English, using unambiguous words, providing context, using terms like you all' often make a better translation.

\section{ANALYSIS}

A multiple language text-to-speech processing apparatus capable of processing a text expressed in multiple languages, and a multiple language text-to-speech processing method.

The multiple language text-to-speech processing apparatus includes a multiple language processing portion receiving multiple language text and dividing the input text into sub-texts according to language and a text-to-speech engine portion having a plurality of text-to-speech engines, one for 
each language, for converting the sub-texts divided by the multiple language processing portion into audio wave data.

The processing apparatus also includes an audio processor for converting the audio wave data converted by the text-to-speech engine portion into an analog audio signal, and a speaker for converting the analog audio signal converted by the audio processor into sound and outputting the sound. Thus, the text expressed in multiple languages, which is common in dictionaries or the Internet, can be properly converted into sound.

Here we use vanilla js with web speech api at back end which process the user data and convert into the audio message.

Speech synthesis is accessed via the Speech Synthesis interface, a text-to-speech component that allows programs to read out their text content (normally via the device's default speech synthesizer .) Different voice types are represented by Speech Synthesis Voice objects, and different parts of text that you want to be spoken are represented by Speech Synthesis Utterance objects. You can get these spoken by passing them to the Speech_Synthesis.speak() method.

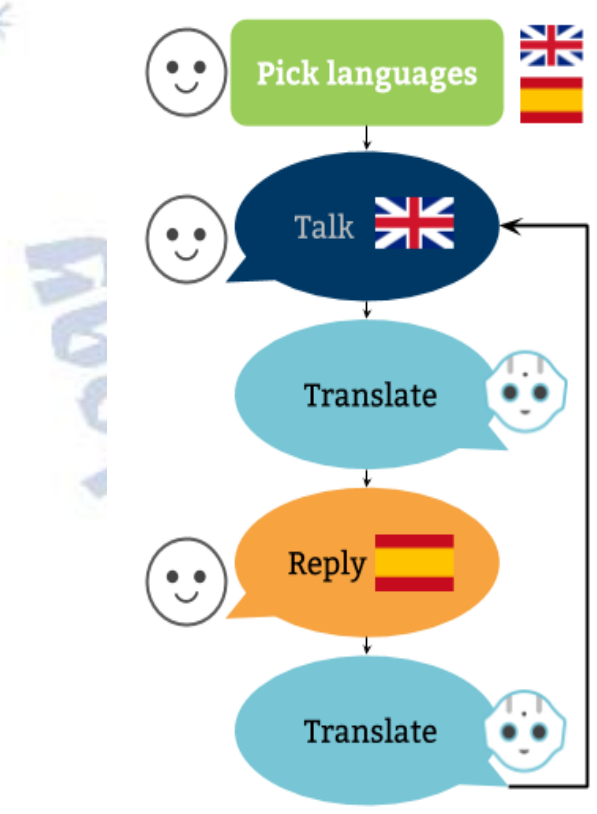

Figure 1 flowchart of development process

\section{RESULTS}

The program will first launch on a browser through which we can access the translator. In the UI you will see different sections like blank text box where we need to give input, select box, pitch and frequency adjustor, and finaly play button.
To run this application first input the text to be converted and then set the rate and pitch fields after this select the language in which you want to convert the text. Finally press play button and the converted text will be speaken by the system.

\section{Speech synthesiser}

Enter some text in the input below and press return or the "play" button to hear it. change voices using the dropdown menu.
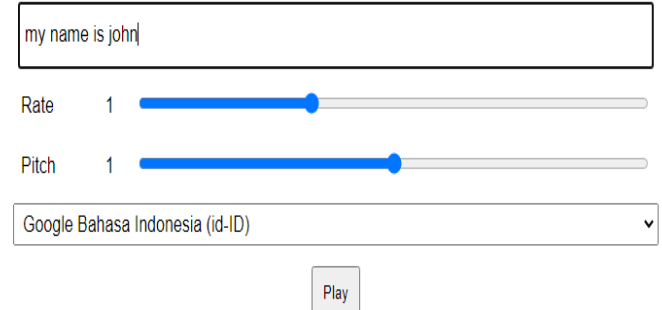

Figure 2 UI of the Project

\section{CONCLUSION}

Throughout the history of mankind different languages comes and go. But now in today's time knowing a single language is not enough and knowing all languages is impossible. So we need a system which can help us in this situation. Therefore this system is very useful for practical use and also easy to use. It erases the language barrier and connect peoples from different regions and background.

\section{REFERENCES}

[1] . Greene Jr, Morgan, et al. "Electronic translator for assisting communications." U.S. Patent No. 6,377,925. 23 Apr. 2002.

[2] Szczepanek, Noah John. "Internet accessed text-to-speech reading assistant." U.S. Patent No. 8,385,525. 26 Feb. 2013. 